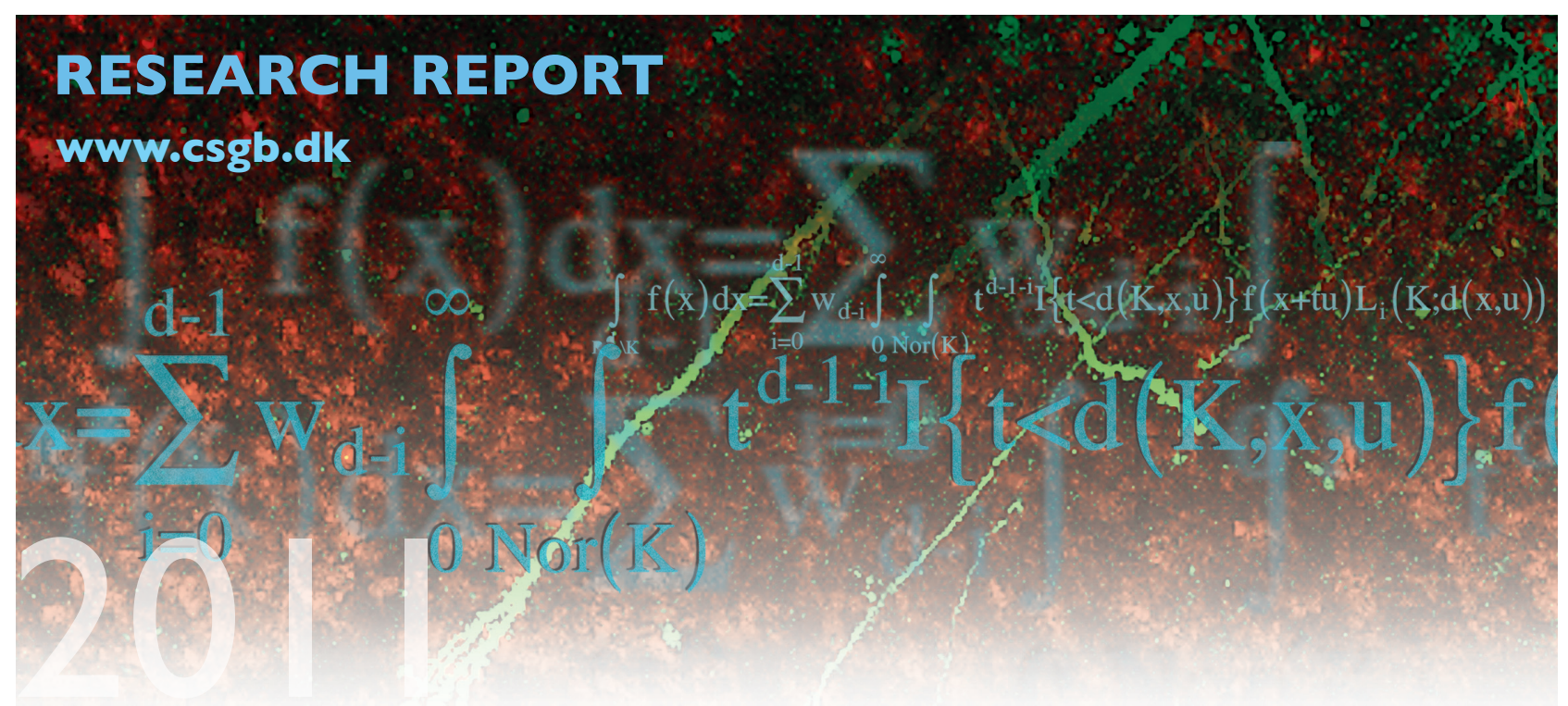

A. Rasmusson, U. Hahn, J.O. Larsen, H.J.G. Gundersen, E.B. Vedel Jensen and J.R. Nyengaard

\title{
The Spatial Rotator
}

No. 06, October 2011 


\title{
The Spatial Rotator
}

\author{
A. Rasmusson ${ }^{1,2,4}$, U. Hahnn ${ }^{2,3}$, J.O. Larsen ${ }^{1,5}$, H.J.G. Gundersen ${ }^{1}$, E.B. \\ Vedel Jensen ${ }^{2,3}$ and J.R. Nyengaard ${ }^{1,2}$ \\ ${ }^{1}$ Stereology and Electron Microscopy, Aarhus University Hospital, Denmark \\ ${ }^{2}$ Centre for Stochastic Geometry and Advanced Bioimaging, Aarhus University, Denmark \\ ${ }^{3}$ Department of Mathematical Sciences, Aarhus University, Denmark \\ ${ }^{4}$ Department of Computer Science, Aarhus University, Denmark \\ ${ }^{5}$ Department of Neuroscience and Pharmacology, , University of Copenhagen, Denmark
}

\begin{abstract}
This paper presents a new local volume estimator, the spatial rotator, which is based on measurements on a virtual 3D probe. The basic design of the probe builds upon the rotator principle which requires only a few manual intersection markings and thus the spatial rotator is fast to use. Since a 3D probe is involved it is expected that the spatial rotator is more efficient than the traditional local volume estimators, the nucleator and the planar rotator, which are based on measurements in a single plane. An extensive simulation study shows that the spatial rotator may be a factor 10 more efficient than the traditional local volume estimators. Furthermore, the spatial rotator can be seen as a derivative of the Cavalieri estimator which does not require randomization of sectioning or viewing direction. The tissue may thus be sectioned in any arbtrary direction where it is easy to identify the specific tissue region under study. In order to use the spatial rotator in practice, however, it is necessary to be able to identify intersection points between cell boundaries and test rays in a series of parallel focal planes, also at the peripheral parts of the cell boundaries. Under- and over-projection phenomena should therefore be negligible or corrected for if the spatial rotator is to be applied.
\end{abstract}

Keywords: Cavalieri, local stereology, particle volume, rotator, virtual probe

\section{Introduction}

It was a major breakthrough in stereology when the local volume estimators: the nucleator (Gundersen, 1988) and the planar rotator (Jensen and Gundersen, 1993), were invented. Dozens of bioscience studies have subsequently been published, using these methods. A few examples are Andersen et al. (2003), Hundahl et al. (2006) and Hosseini-Sharifabad and Nyengaard (2007).

Corresponding author: A. Rasmusson, alras@cs.au.dk 
The local volume estimators are typically used in light microscopy of transparent biological tissue where it is possible to place the focal plane at different depths within thick tissue sections $(>20 \mu \mathrm{m})$. The first step is to use the optical disector (Sterio, 1984; Gundersen, 1986) for number-weighted sampling of particles. The second step is to estimate the volume of each sampled particle, using measurements on a local probe that passes through a unique point of the particle. The vast majority of currently developed probes have been designed such that they are contained in the focal plane passing through the unique point of the sampled particle.

This is also true for the recent pivotal estimators based on the invariator principle, see Cruz-Orive (2008) and the accompanying papers Cruz-Orive (2009) and CruzOrive et al. (2010). Unfortunately this ignores the 3D information about the particles, available in thick tissue sections. This observation was the background for the development of the optical rotator in Tandrup et al. (1997). Here, the available $3 \mathrm{D}$ information was utilized in a probe, consisting of test lines in several planes at different optical depths in relation to the sampled particle. Unfortunately, the extra manual workload of marking intersection points with more test lines made it too time consuming and it has thus rarely been used in practice. However, it did show that it is beneficial to use extra information from the entire particle.

The main challenge for the practical implementation of the local volume estimators is that they require generation of isotropic random test lines which can be done either via vertical sections (Baddeley et al., 1986) or isotropic sections (Mattfeldt et al., 1985; Nyengaard and Gundersen, 1992). The use of vertical and isotropic sections in brain tissue, other complex tissues and archival tissue may not be possible or practical due to the loss of orientation and identification of specific tissue regions.

There is therefore a need for developing a local volume estimator which does not require vertical or isotropic sections. The spatial rotator, developed in the present paper, is based upon the idea of avoiding vertical or isotropic sections and to utilize the extra 3D information available in the thick sections.

The composition of the paper is as follows. In Section 2, the estimation principle of the spatial rotator is described and the statistical properties of the spatial rotator are discussed. Section 3 contains a thorough simulation study while various aspects of the practical implementation of the spatial rotator are studied in Section 4. Section 5 contains a discussion. The derivation of the estimation principle of the spatial rotator is deferred to an Appendix.

\section{The spatial rotator}

\section{$2.1 \quad$ Estimation principle}

The spatial rotator is a design-based method to estimate the volume of a particle from measurements on systematic test rays. These test rays are perpendicular to an axis chosen in advance, the so-called local arbitrary vertical axis (LAVA). In this subsection, we introduce the theoretical sampling principle; possible implementations are described later.

- First, a LAVA through or close to the particle is chosen, and the endpoints 
of the projection of the particle onto the LAVA are determined. One of the endpoints serves as reference point for the test rays later. Let $L_{\text {proj }}$ be the projection length.

- Next, $n$ test rays, perpendicular to the LAVA, are systematic uniform randomly sampled (SURS) by sampling their starting points SURS on the LAVA and their directions SURS on $[0,2 \pi]$, see Figure 1 . The starting point of the $i$ th ray is given by its distance $p_{i}$ to the reference endpoint of the LAVA. The position $p_{1}$ of the first ray is drawn uniform randomly in the interval $[0, d]$, where $d=L_{\text {proj }} / n$. For the remaining test rays, $p_{i}=p_{1}+(i-1) d$. Since the test rays are perpendicular to the (vertical) LAVA, their directions are determined by planar angles to some given horizontal reference axis. The angle $\alpha_{1}$ specifying the direction of the first test ray is drawn uniform randomly in $[0, \vartheta]$ where $\vartheta=2 \pi / n$. The direction of the $i$ th test ray is given by $\alpha_{i}=\alpha_{1}+(i-1) \vartheta$.

- Finally, on each test ray, the distances from the LAVA to the intersection points with the particle are measured. Let $\ell_{i k}$ denote the $k$-th intersection distance on the $i$-th ray, starting with the longest (i.e., $\ell_{i 1}>\ell_{i 2} \ldots$ ). Calculate the so-called squared ray distance

$$
\ell_{i}^{2}=\sum_{k} \ell_{i k}^{2} \cdot(-1)^{k+1}
$$

and obtain the volume estimator by adding the results for all test rays and scaling with $\pi$ and the distance $d$ between test rays:

$$
\hat{V}=d \pi \sum_{i=1}^{n} \ell_{i}^{2} .
$$

Note that the squared ray distance in Gundersen (1988) or Jensen and Gundersen (1993) has a different form since there the $\ell_{i k}$ 's were numbered starting with the smallest distance.

\subsection{Relation to the Cavalieri estimator}

The spatial rotator can be seen as a derivative of the Cavalieri estimator, cf. Baddeley and Jensen (2005, pp. 155-158) and references therein. The Cavalieri estimator uses profile areas measured on an exhaustive stack of $n$ serial parallel sections with uniform random position. Let $A_{i}$ denote the area of the particle profile on the $i$-th section, and let $d$ be the distance between sections, then

$$
\hat{V}_{\text {Caval }}=d \sum_{i=1}^{n} A_{i}
$$

is an unbiased estimator for the particle volume. This still holds true if the area $A_{i}$ is replaced by an unbiased estimator $\hat{A}_{i}$ of $A_{i}$. Often, the area is estimated by counting points on a test grid or measuring intersections with parallel lines (Baddeley and Jensen, 2005, pp. 158-162). The spatial rotator can be considered as a 


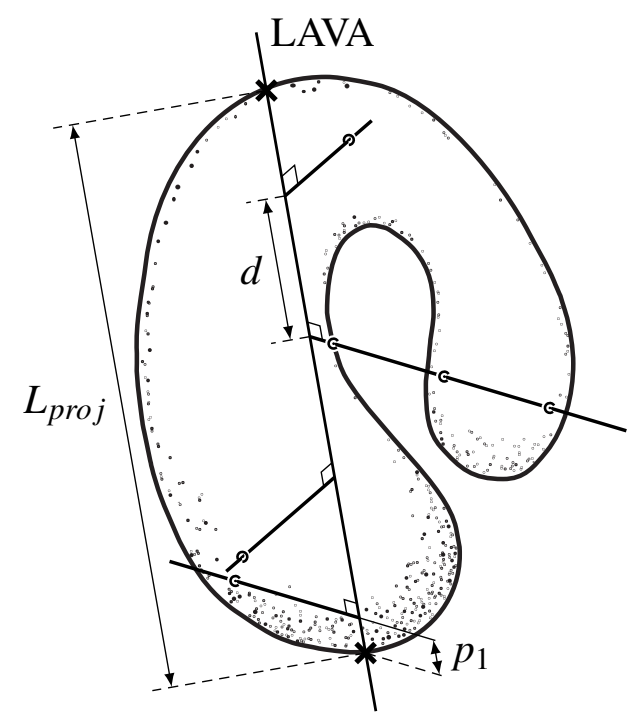

Figure 1: Particle with spatial rotator probe, consisting of $n=4$ systematic test rays perpendicular to the local arbitrary vertical axis (LAVA). In this example, the third ray (counted from below) intersects the particle three times while the other rays only intersect once.

Cavalieri estimator using another method to estimate planar areas, the two dimensional nucleator, see Gundersen (1988) and Jensen (1998, pp. 95-96). This is a local stereological estimator based on measuring intersection distances of test rays that emanate from a fixed point. It has the form

$$
\hat{A}_{i}=\pi \ell_{i}^{2},
$$

where $\ell_{i}^{2}$ is calculated following (2.1). Systematic sampling of section planes for Cavalieri volume estimation and of the test ray positions for the spatial rotator correspond to each other, since every ray is contained in a unique plane perpendicular to the LAVA, as illustrated in Figure 2. Thus, the spatial rotator can be interpreted as a Cavalieri estimator, where the individual estimators $\hat{A}_{1}, \hat{A}_{2}, \ldots$ of the intersection areas are not independent, but linked to each other by the systematic sampling of the test ray directions.

\subsection{Statistical properties}

Unbiasedness of the spatial rotator follows directly from the interpretation as a derivative of the Cavalieri estimator and from the unbiasedness of the two dimen- 


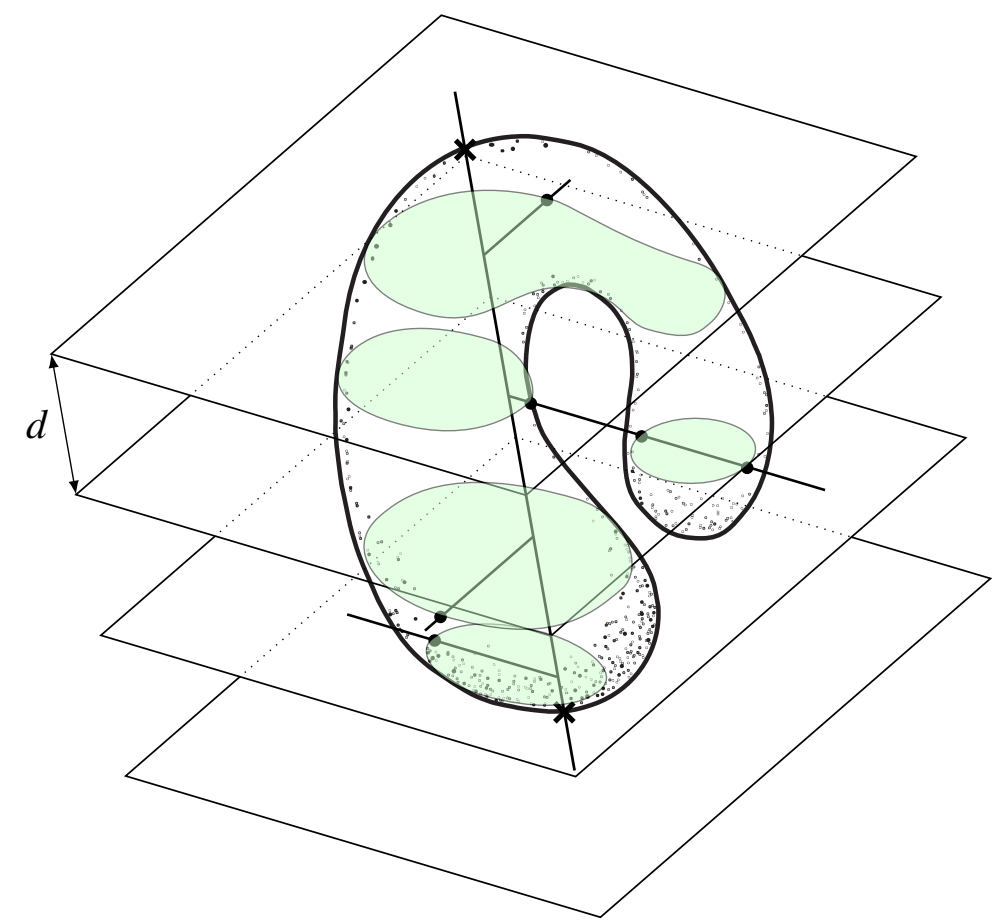

Figure 2: Particle intersected with $n=4$ parallel planes perpendicular to the LAVA depicted in Figure 1. Section profiles are drawn together with the test rays of the corresponding spatial rotator.

sional nucleator. We have

$$
\begin{aligned}
\mathrm{E} \hat{V} & =\mathrm{E}\left(\mathrm{E}\left(\hat{V} \mid p_{1}\right)\right) \\
& =\mathrm{E}\left(\mathrm{E}\left(d \pi \sum_{i=1}^{n} \ell_{i}^{2} \mid p_{1}\right)\right) \\
& =\mathrm{E}\left(d \sum_{i=1}^{n} \mathrm{E}\left(\pi \ell_{i}^{2} \mid p_{1}\right)\right) \\
& =\mathrm{E}\left(d \sum_{i=1}^{n} A_{i}\right)=\mathrm{E} \hat{V}_{\text {Caval }},
\end{aligned}
$$

where $A_{i}$ is the area of the particle profile on a section perpendicular to the LAVA, with distance $p_{i}=p_{1}+(i-1) d$ to the reference point on the LAVA.

The estimation variance depends on the particle shape, the position and direction of the LAVA, and on the number $n$ of test lines. Consider a given particle and a given LAVA and fix $n$. Then the estimate $\hat{V}$ depends on the starting point $p_{1}$ and on the starting angle $\alpha_{1}$, and the variance can be decomposed as follows:

$$
\begin{aligned}
\operatorname{Var} \hat{V} & =\operatorname{Var}\left(\mathrm{E}\left(\hat{V} \mid p_{1}\right)\right)+\mathrm{E} \operatorname{Var}\left(\hat{V} \mid p_{1}\right) \\
& =\operatorname{Var}\left(\hat{V}_{\text {Caval }}\right)+\mathrm{E} \operatorname{Var}\left(\hat{V} \mid p_{1}\right) .
\end{aligned}
$$

We will call the first term, $\operatorname{Var}\left(\hat{V}_{\text {Caval }}\right)$, the Cavalieri variance in the following. $\operatorname{Var}\left(\hat{V}_{\text {Caval }}\right)$ depends on the shape of the particle and has been the subject of many thorough studies, cf. Baddeley and Jensen (2005, Chapter 13) and references therein. 


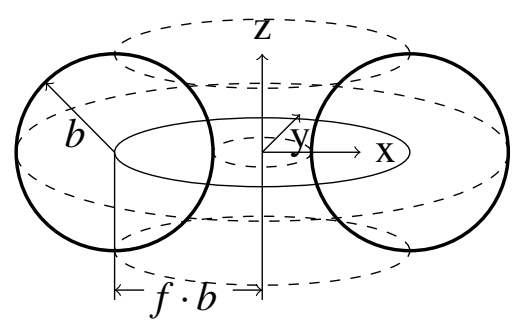

Figure 3: A rotus can be generated by rotating a circle with radius $b>0$ around a fixed axis a distance $f \cdot b$ from this axis where $f \geq-1$. For $f>1$, an ordinary torus is obtained.

The second term is the variance due to the randomization of $\alpha_{1}$. Writing $A_{\text {tot }}=$ $\sum_{i} A_{i}$ for the total area of section profiles of the particle with the stack of serial Cavalieri sections, we may interpret $\operatorname{Var}\left(\hat{V} \mid p_{1}\right) / d^{2}$ as the variance of estimating $A_{\text {tot }}$ with an estimator related to the two dimensional nucleator, using a system of $n$ test rays. We will therefore refer to $\mathrm{E} \operatorname{Var}\left(\hat{V} \mid p_{1}\right)$ as the nucleator variance. The nucleator variance depends on the shape of the profiles and on the position of the starting point of the test rays on the Cavalieri sections, i.e. the trace of the LAVA, with respect to the profiles. The variance is minimized if this point lies centrally in the profiles, as illustrated in Gundersen (1988, Figure 5). In practice this will hardly be fulfilled, in particular not for non-convex particles as the example shown in Figure 1.

\section{Simulation study}

In this section we study by simulation how the efficiency of the spatial rotator is affected by the choice of the position and orientation of the LAVA relative to the particle as well as the choice of angular spacings of the rays emanating perpendicularly from the LAVA. We also investigate the gain in efficiency by using the spatial rotator compared to its planar analogue, the planar rotator (Jensen and Gundersen, 1993).

\subsection{Particles}

A rotus can be generated in the same manner as a torus by rotating a circle with radius $b>0$ around a fixed axis a distance $f \cdot b$ from this axis, see Figure 3 . In the torus case $f>1$, but the rotus allows $f>-1$ such that the rotating circles may overlap. For $f \leq 0$, the generated rotus will be convex, for $f=0$ the rotus is a perfect sphere. A great shape variability is thereby possible within the class of rotus shaped particles.

In the simulations to be presented below, particles of five different rotus shapes were used, see Figure 4 . The size parameter $b$ was chosen such that all rotus particles had volume 1 .

\subsection{Probes}

The specification of the spatial rotator needs, besides the choice of number of test rays, a choice of orientation and position of the LAVA and a scheme for choosing 

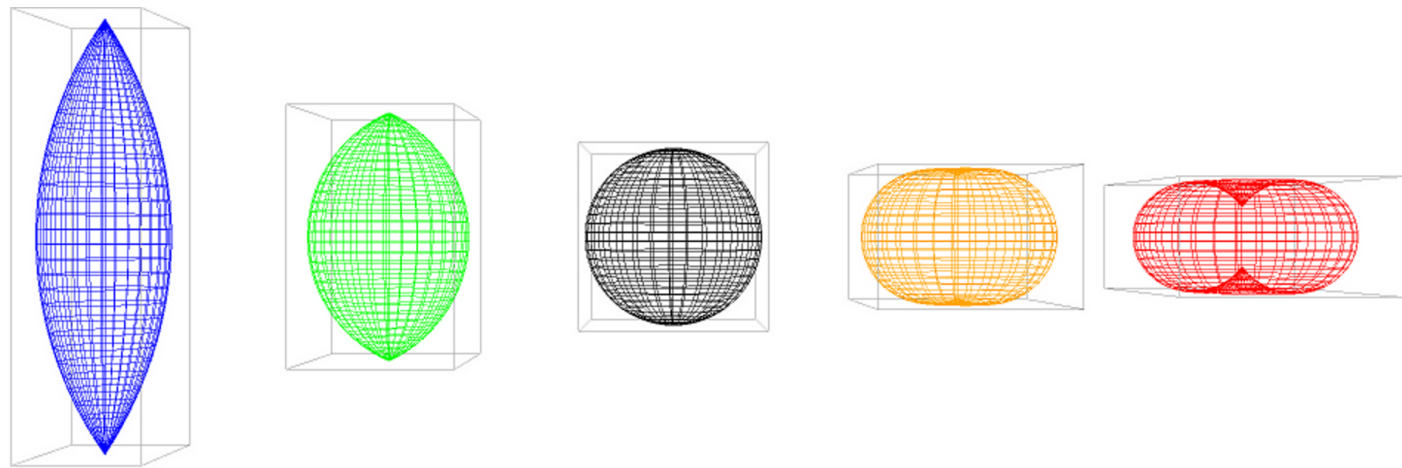

Figure 4: Silhouttes of the five rotus particles used in the simulation study, The size parameter $b$ was chosen such that all rotus particles had volume 1. The leftmost particle has shape parameter $f=-0.85$, the particle in the middle is a perfect sphere and the rightmost has $f=0.85$.

the angular spacing between the test rays.

\subsubsection{Choice of LAVA}

Originally, it was believed that the optimal choice of LAVA was the one that gave the longest particle intercept. For the rotus particles used in the simulation study with $f \leq 0$ this choice corresponds to the axis of symmetry while for rotus particles with $f \geq 0$ this choice corresponds to an axis perpendular to the symmetry axis, i.e. an axis of asymmetry. In the simulation study, the efficiency of the spatial rotator obtained, using these two choices of LAVA, was compared with that obtained for an isotropically oriented LAVA where no information about particle shape is used. LAVAs with an angular offset of $\frac{\pi}{8}$ and $\frac{\pi}{16}$ radians, respectively, from either the axis of symmetry or the axis of asymmetry were also considered in the simulation study.

The LAVAs were chosen to pass through a uniform random point inside a centrally placed subregion of the rotus particle, obtained by rescaling. By varying the scale factor it was easy to vary the subregion for any complex shape of the rotus particle. The impact due to position was investigated using the exact centre of the rotus particle and uniform random points inside subregions with a $25 \%, 50 \%$ and $99 \%$ scale factor.

A total of 28 different choices of LAVA were thus studied:

- 12 choices with LAVA passing through a uniform random point in a subregion with scale factor $0 \%$ (exact centre), $25 \%, 50 \%$ and $99 \%$, respectively, and with angle $0, \frac{\pi}{8}$ and $\frac{\pi}{16}$ radians, respectively, to the axis of symmetry

- 12 choices obtained as above, but with the symmetry axis replaced by the asymmetry axis

- 4 choices with LAVA passing through a uniform random point in a subregion with scale factor $0 \%, 25 \%, 50 \%$ and $99 \%$, respectively, and with isotropic direction

Each choice of LAVA was simulated with $n=1,2,3,4,5,6,8,9,10,12,15,18,20$ test rays, respectively. 


\subsubsection{Angular Spacing}

A simple way of choosing the angular spacing is to take one full revolution divided by the number of test rays. This is the approach described in Section 2 . The angle of the first test ray $\alpha_{1}$ is uniform random in $[0, \vartheta]$ where $\vartheta=2 \pi / n$. The angle of the $i$ th test ray is given by $\alpha_{i}=\alpha_{1}+(i-1) \vartheta$. It might, however, be an advantage to use a scheme where more than one revolution is involved, thereby possibly obtaining a more complete sampling of the particle under study. In the simulation study, we therefore also used a two-revolution scheme with

$$
\vartheta= \begin{cases}4 \pi / n, & n \text { odd } \\ 4 \pi /(n-1), & n \text { even }\end{cases}
$$

For comparison, we in addition considered independent and uniform random angles $\alpha_{i}$ in $[0,2 \pi]$.

\subsection{Main results from the simulation study}

For a given particle shape, determined by the rotus shape parameter $f$, the precision of the spatial rotator was found to depend both on the orientation of the LAVA and the point that determines the position of the LAVA. For the rotus particles considered in this study, the variance was minimized if the LAVA coincided with the axis of symmetry. Note that this axis is not always the longest axis passing through the particle under study. The squared coefficient of error $\left(\mathrm{CE}^{2}=\right.$ variance divided by squared mean) obtainable under these ideal conditions is depicted in the left part of Figure 5, as a function of the number $n$ of test rays used in the estimator. The $\mathrm{CE}^{2}$ increases as soon as the "nucleus" through which the LAVA passes is not situated in the centre of the particle. In that case, the simulations showed that deviation of the LAVA orientation from the ideal symmetry axis direction is of very little influence on the efficiency of the spatial rotator. The middle part of Figure 5 shows the spatial rotator $\mathrm{CE}^{2}$ for a LAVA positioned in a uniform random point chosen within a $50 \%$ subregion and with isotropic random direction. For comparison, the $\mathrm{CE}^{2}$ of the isotropic planar rotator with corresponding placement of the axis reference point is shown in the right part of the figure. Note the quite strong difference to the variance prediction for the planar rotator in the paper of Jensen and Gundersen (1993), which is due to the fact that the present simulation study also took the randomization of the section plane into account. Note also that the isotropic spatial rotator is more efficient than the isotropic planar rotator if at least 4 or 5 test rays are used. For $n=10$ test rays, the variance of the isotropic spatial rotator is approximately 10 times smaller than that of the planar rotator.

Table 1 gives an impression of how the precision of the spatial rotator depends on the position of the LAVA. It lists the $\mathrm{CE}$ (the square root of $\mathrm{CE}^{2}$ ) of the spatial rotator with isotropic LAVA for the rotus shapes included in this study and $n=6$ test rays. Note that a CE less than 0.1 is obtainable with 6 test rays if the particle is not too elongated and the point through which the spatial rotator passes is not too far from the centre of the particle.

In order to find out whether the two-revolution angular spacing scheme is advantageous compared to the one-revolution scheme and i.i.d. sampling (independent 

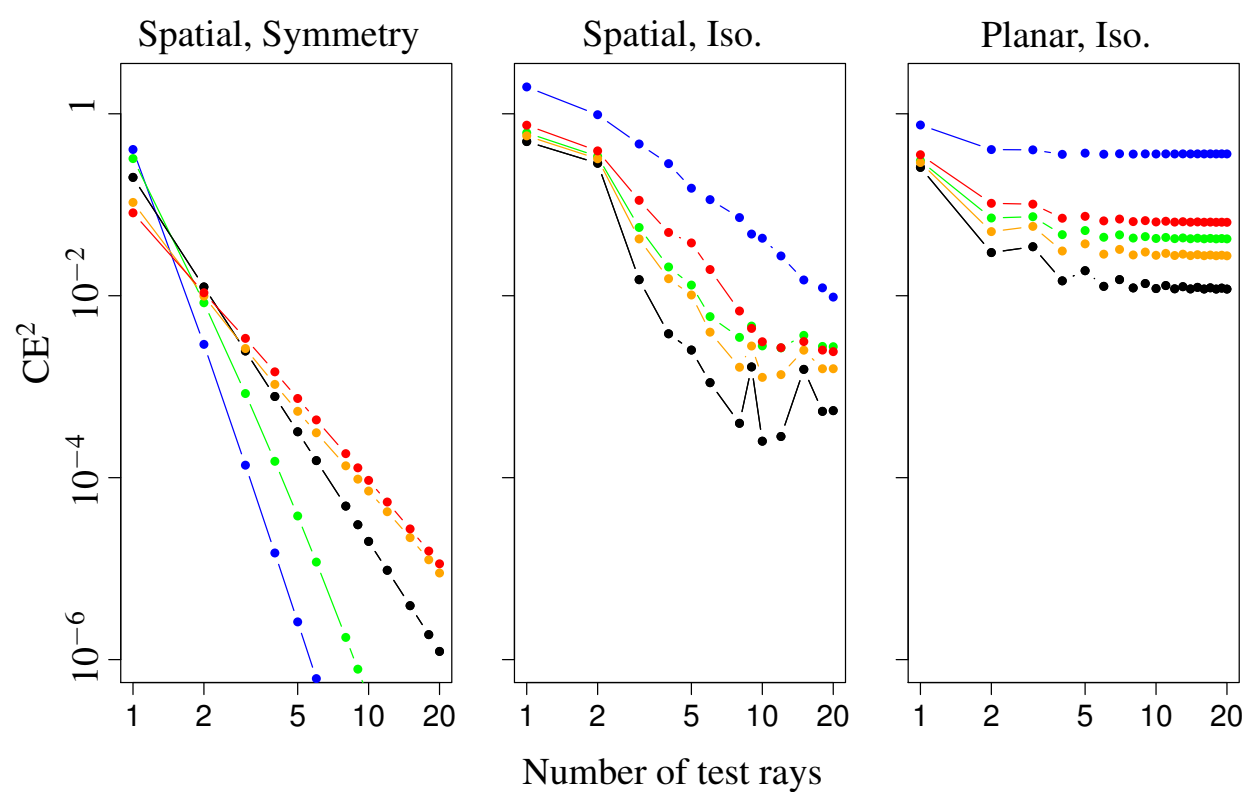

Figure 5: $\mathrm{CE}^{2}$ of spatial rotator and isotropic planar rotator as a function of the number $n$ of test rays. Left panel: ideal case, LAVA identical to the axis of symmetry. Middle panel: isotropic LAVA, positioned uniform randomly within a central $50 \%$ subregion of the particle, as explained in Section 3.2.1. Right panel: $\mathrm{CE}^{2}$ of the isotropic planar rotator, with nucleus in a central $50 \%$ region. Colours reflect the different rotus particles and correspond to Figure 4.

Table 1: Coefficient of error of a spatial rotator with $n=6$ test rays. Isotropic LAVA positioned within central regions of various extent. For further details, see the text.

\begin{tabular}{lcccc}
\hline Particle & $0 \%$ & $25 \%$ & $50 \%$ & $99 \%$ \\
\hline Strongly elong. & 0.250 & 0.277 & 0.337 & 0.517 \\
Slightly elong. & 0.056 & 0.064 & 0.077 & 0.136 \\
Spherical & 0.012 & 0.019 & 0.033 & 0.074 \\
Slightly flat & 0.042 & 0.048 & 0.063 & 0.115 \\
Strongly flat & 0.099 & 0.107 & 0.139 & 0.210 \\
\hline
\end{tabular}




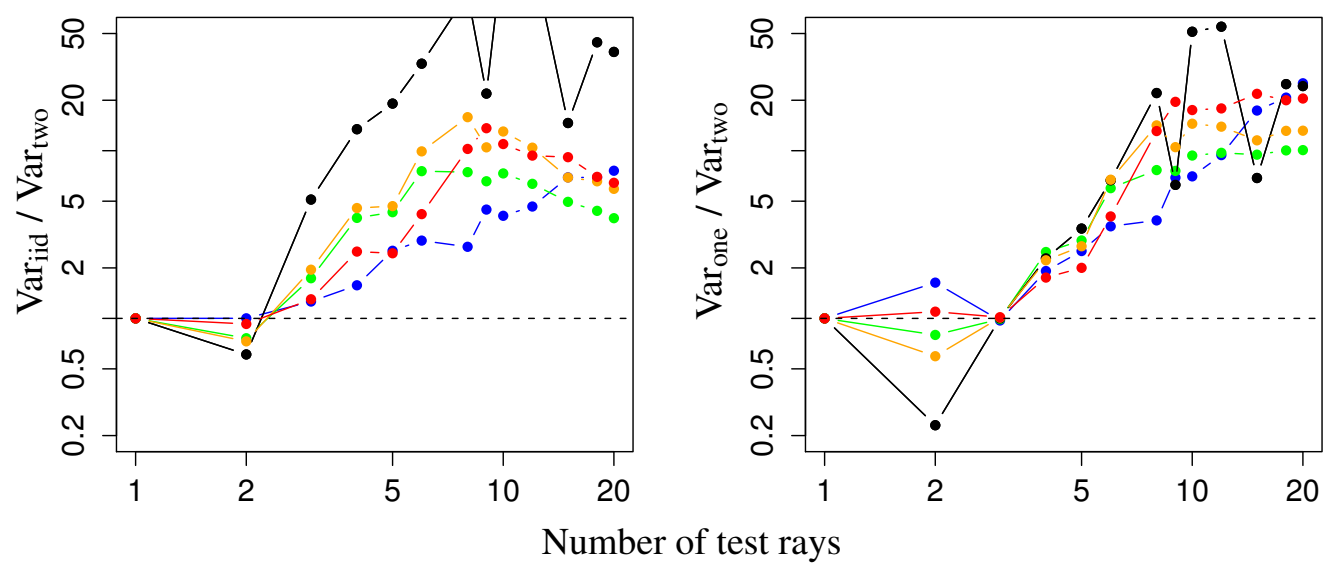

Figure 6: Relative efficiency of the spatial rotator based on the two-revolution scheme of choosing the angular spacings vs the spatial rotator based on i.i.d. angular spacings (left part) and the one-revolution scheme (right part), respectively. The LAVA is isotropic and passes through a uniform random point in a subregion of the rotus particle with scale factor $25 \%$. Colours reflect different rotus particles and correspond to Figure 4.

and identically distributed uniform random ray angles), the variance $\operatorname{Var}_{\text {two }}$ of the spatial rotator based on the two-revolution angular scheme was compared to the variance $\operatorname{Var}_{\text {one }}$ obtained, using the simple one-revolution scheme, and the variance $\operatorname{Var}_{\text {iid }}$ obtained, using i.i.d. angular spacings. Figure 6 shows the relative efficiencies $\operatorname{Var}_{\text {iid }} / \operatorname{Var}_{\text {two }}$ and $\operatorname{Var}_{\text {one }} / \operatorname{Var}_{\text {two }}$ for the spatial rotator with isotropic LAVA passing through a uniform random point in a subregion of the rotus particle with scale factor $25 \%$. The depicted example is typical for other choices of the LAVA. Note that the one- and two-revolution schemes coincide for $n=3$ test rays. For numbers of test rays important in practice, $n>3$, say, the two-revolution scheme turned out to be superior to both alternative schemes of choosing the angular spacings - for moderate numbers of test rays around $n=10$, the two-revolution scheme is about ten times as efficient as the one-revolution scheme, and for the practical important number $n=5$, it still halves the variance. The advantage over independent sampling starts to decrease for numbers of test rays $n$ larger than 10 .

As mentioned in Section 2.3, the variance of the spatial rotator can be decomposed into the Cavalieri variance related to the randomization of the test ray end points on the LAVA and the nucleator variance related to the randomization of the angles of the test rays emanating perpendicularly from the LAVA. Figure 7 shows the two variance components as a function of the number of test rays for the cases of a LAVA parallel to the symmetry axis (left) and for an isotropic LAVA (right).

While there is a clear difference between the Cavalieri variances for the different rotus particles if the axis is parallel to the symmetry axis, these differences vanish if the axis is isotropic. Similar results have been obtained for other LAVAs that are not parallel to the symmetry axis of the particle. This behaviour can be explained by the fact that a fixed anisotropic particle "looks" isotropic to an isotropic axis. A converse effect can be observed for the nucleator variance component. Note also that the plots in Figure 7 indicate that there is an asymptotic saturation behaviour in the nucleator variance with increasing number of test rays. This can be explained by the 

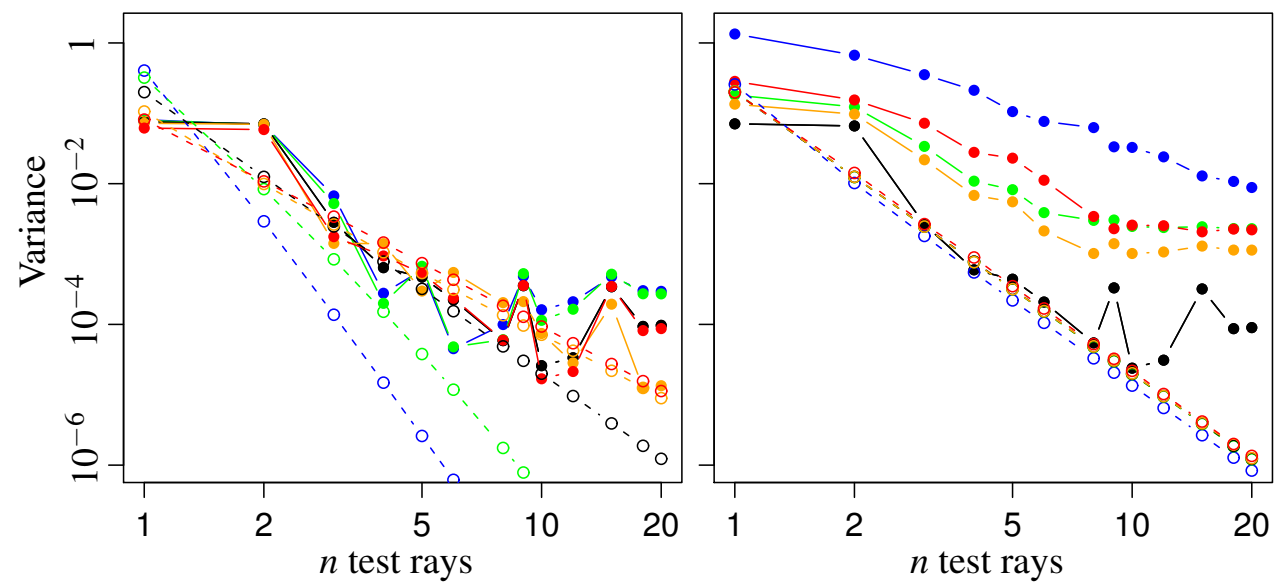

Figure 7: Cavalieri variance (hollow dots, dashed lines) and nucleator variance (solid dots, solid lines) for a LAVA parallel to the symmetry axis (left) and an isotropic LAVA (right). In both cases, the LAVA passes through a uniform random point, chosen in a subregion with scale factor $25 \%$. The two-revolution angular spacing scheme was used. Colours reflect the different rotus particles and correspond to Figure 4.

fact that the systematic sampling scheme of the spatial rotator is concentrated on a one-dimensional subset of the two-dimensional set of (position, angle) determining the test rays. Note also that the dominating component of variance for the spatial rotator is the nucleator variance.

\section{On the practical implementation of the spatial rotator}

\subsection{Arbitrary LAVA}

In cases where the option of randomizing the LAVA is not available, for instance because randomization will cause loss of possibility of identifying the specific tissue region under study, the traditional local volume estimators, the nucleator and the planar rotator, cannot be used. In this situation, the spatial rotator offers a valid alternative since its LAVA can be chosen arbitrarily.

If local sectional areas are available, the Cavalieri estimator might be preferred to the spatial rotator because of lower variance, see Figure 7 . Whether the Cavalieri estimator is to be preferred depends on (1) the practical problems involved in determining the sectional areas compared to the intersection points and (2) the size of the variance reduction compared to the variance in the true particle volume distribution.

The spatial rotator involves the determination of intersection points between test rays and particle boundaries. Usually, a test ray can only be indirectly observed via its intersections with each of a series of parallel focal planes. If the test ray is not parallel to the common direction of the focal planes, the intersection between the test ray and a focal plane is a single point. The only way to determine when such a test ray intersects the particle boundary is to observe how this intersection 


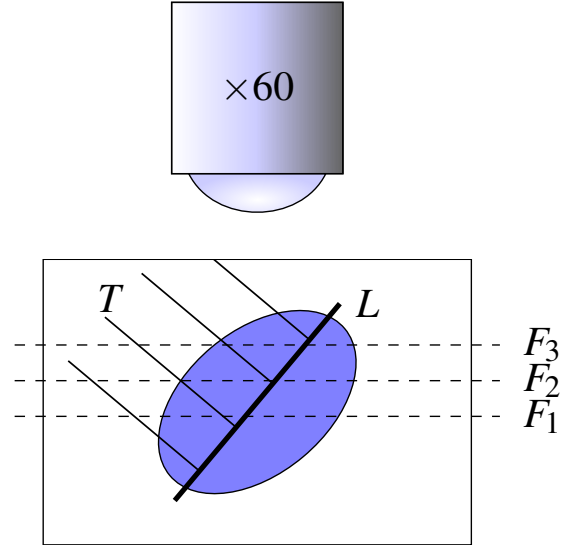

(a)
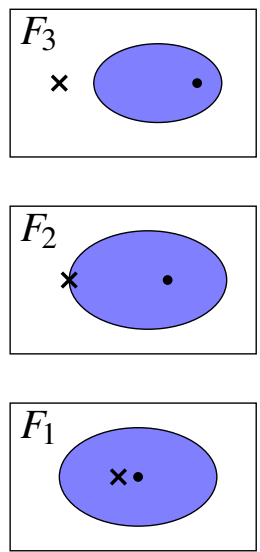

(b)

Figure 8: Determination of intersection points between test rays and particle boundary. (a) 2D illustration of a spatial rotator with LAVA $L$ with arbitrary orientation relative to the series of focal planes $F_{1}, F_{2}$ and $F_{3}$. A particular test ray is denoted $T$. (b) On $F_{1}, F_{2}$ and $F_{3}$, intersection points between the focal planes and the test ray is indicated by $\times$. In $F_{2}$, the intersection point lies at the particle boundary. The intersection points between the focal planes and the LAVA $L$ are shown as

point moves when moving the focal plane up and down. When the intersection point moves from one side of the particle boundary to the other, the intersection point has reached the particle boundary, see Figure 8.

This procedure may be very time consuming and, accordingly, have a huge impact on how quickly an operator can mark the intersection points between all the test rays and the particle boundary. For this reason, it is advisable to choose the LAVA perpendicular to the focal plane. Then, a test ray will be fully contained in one focal plane and the marking will be as simple as for other well-known probes, see Figure 9.

When using the spatial rotator it is important to be able to determine the top and bottom of a particle accurately in order to place test rays inside this interval and subsequently to determine the intersection points between the particle boundaries and the test rays accurately. It is in particular important when the LAVA is chosen arbitrarily since in this case no other local estimator of volume is available (they require randomization of the LAVA) and a possible bias introduced by over- and under-projection cannot be corrected for by comparison with other unbiased local volume estimators.

There is another version of the spatial rotator that does not require exact knowledge of the top and bottom of the particle. Here, the distance $d$ between endpoints of neighbour test rays may be chosen without precise knowledge of the projection of the particle on the LAVA. The spatial rotator estimator is still of the form (2.2) but since the projection length of the particle need no longer be an integer multiple of $d$, the number $n$ of test rays hitting the particle may vary from one realization of the spatial rotator to the other. This version of the spatial rotator avoids the problem that the top and bottom of the particle should be known but it is still needed to determine intersection points between test rays and particle boundaries at peripheral parts of the particle where its boundary may be fuzzy due to underor over-projection. 


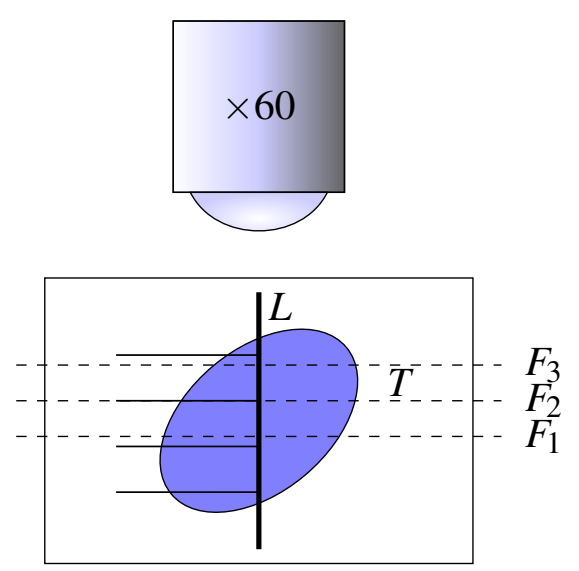

(a)

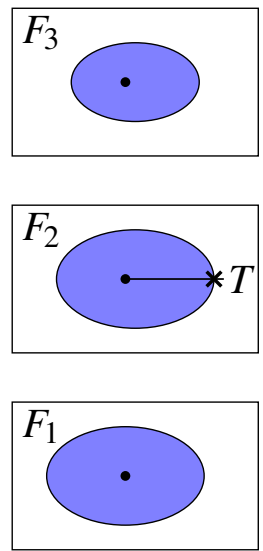

(b)

Figure 9: Determination of intersection points between test rays and particle boundary. (a) 2D illustration of a spatial rotator with LAVA $L$ perpendicular to the series of focal planes $F_{1}, F_{2}$ and $F_{3}$. A particular test ray is denoted $T$. (b) The test ray $T$ is fully contained in $F_{2}$ and here it is easy to identify the intersection point $\times$ between $T$ and the particle boundary. The intersection points between the focal planes and the LAVA $L$ are shown as

\subsection{Randomized LAVA}

If it is possible to randomize the LAVA (placing it either vertical random or isotropic random) and still identify the structure under study, both the planar and the spatial rotator can in principle be used as local volume estimators. The importance of a possible bias of the spatial rotator, introduced by under- and over-projection at the peripheral parts of the particles, can then be assessed by a comparison with the planar rotator. This local volume estimator will be virtually unbiased since it only uses information from the centre of the particle where under- and over-projection are not important. Below we give a typical example of this situation.

\subsubsection{Materials}

The study complied with the Danish regulations for care and use of experimental animals and was approved by the Danish Animal Experiments Inspectorate. One Wistar rat from the animal facility at Aarhus University Hospital Risskov was transcardially perfusion-fixed in deep pentobarbital anesthesia (Mebumal SAD, $50 \mathrm{mg} / \mathrm{mL}$ ). Icecold isotonic saline perfusion was followed by perfusion of $4 \%$ phosphate- buffered paraformaldehyde. The right or left randomly chosen hippocampus was cut into $2 \mathrm{~mm}$ slabs, embedded in the isector (Nyengaard and Gundersen, 1992) in agar and next in glycolmethacrylate (Technovit 7100 kit, Kulzer Histo-Technik, Heraeus Kulzer, Wehrheim, Germany). An isotropic, uniform random 40 um thick section was cut from the centre of each hippocampus block using a Ralph glass knife mounted on a calibrated microtome (Supercut, Reichert-Jung) and stained with a modified Giemsa stain. An Olympus BX51 microscope equipped with a 60x Olympus oil lens (S-Plan, NA 1.40), a MT12 microcator (Heidenhain, Germany), a motorized specimen stage (Merzhauser, Germany), and an Olympus DP70 digital camera connected 
to a Dell PC (Intel Core 2 Duo, $2500 \mathrm{MHz}$ ) with 4 GB memory and a GForce 8800 GPU with 512 MB RAM were used.

\subsubsection{Sampling $z$-stacks of digital images}

Systematic uniform random sampling of fields of view of the hippocampal subregion subiculum was performed, and two-dimensional unbiased counting frames were superimposed onto the images. The disector height was set to $30 \mu \mathrm{m}$ and neurons were sampled when the nucleoli came into focus in the optical plane (z-axis) and were sampled by the 2D counting frame. 238 neurons in subiculum were sampled by the optical disector (Gundersen, 1986). For each sampled field of view, a $z$-stack of digital images of sections with thickness $1 \mu \mathrm{m}$ through the whole thick section was obtained. Image recording, disector-sampling of neurons and estimation of their volume using the planar rotator was done using the newCAST software (ver. 3.4, Visiopharm, Denmark). The implementation of the spatial rotator was performed using $\mathrm{C}++$ programming language (gcc V.4.4.4 for the linux platform and MSVC $2005 / 2008$ for Win 7 ). For practical application of the probe on the recorded $z$-stacks both probe and focal planes were rendered using OpenGL v.1.3. The boost random library (v. 1.42) was used for isotropic and structural uniform random sampling for both the application of the probe and the simulations.

\subsubsection{Results}

In this example, it was very difficult to determine the top and bottom of the cells. Various methods were tried out, see the Discussion. The method that worked best was based on determination of the largest radius $r$ from the nucleolus to the cell boundary, measured in the focal plane through the nucleolus, cf. Figure 10a. The top and bottom of the cell were placed $r$ above and below the nucleolus. This procedure will work well if the cells are fairly spherical. A possible bias of the spatial rotator may be introduced by this procedure, but its magnitude can be checked by comparing with the planar rotator. Marking of intersection points between cell boundary and test rays is shown in Figure 10b-f.

The spatial rotator and the planar rotator were determined, using the abovementioned software tools, for 238 neurons in the subiculum. In Figure 11, the spatial rotator volume estimates are plotted against the planar rotator volume estimates. For comparison, the identity line is also shown in Figure 11. A small bias (10\%) can be detected. More precisely, the regression line through the origin obtained by least squares fitting has slope 1.08. The coefficient of error was of the same size for planar and spatial rotator volume estimates, being in the order of magnitude of $\mathrm{CE}^{2} \approx 0.25$. The size of the squared coefficient of error is probably mainly due to biological variation. In this situation, the planar rotator is to be preferred. 


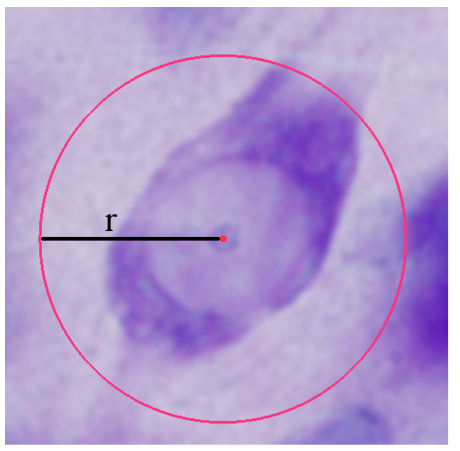

(a)

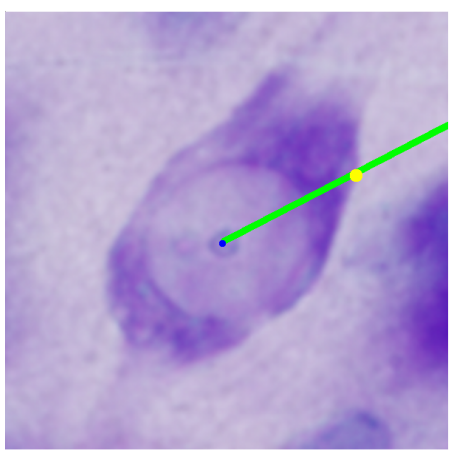

(d)

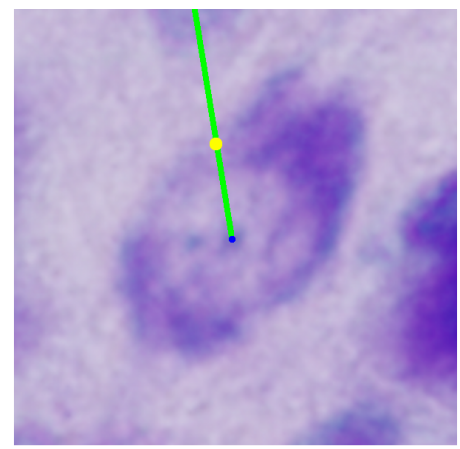

(b)

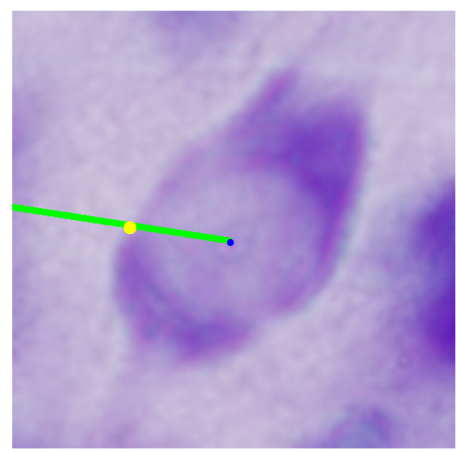

(e)

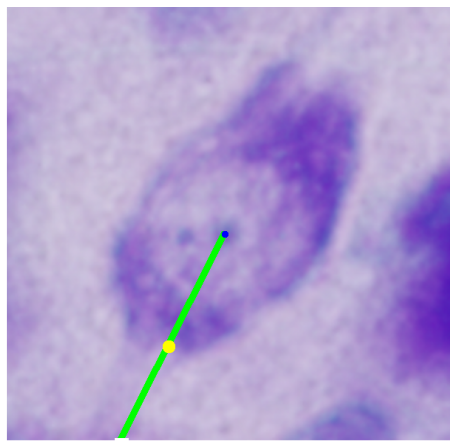

(c)

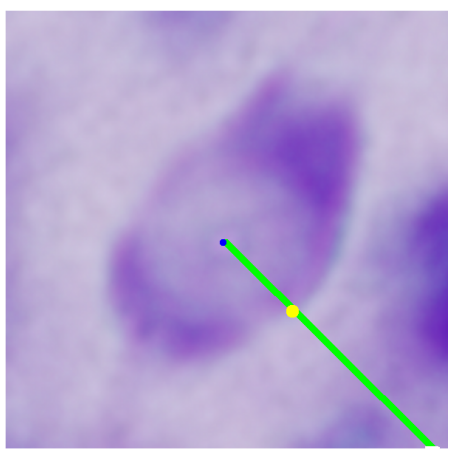

(f)

Figure 10: Illustration of the practical implementation of the spatial rotator. (a) The largest radius $r$ from the nucleolus to the cell boundary in the focal plane through the nucleolus is indicated. The top and bottom of the cell were placed $r$ above and below the nucleolus. (b)-(f) Marking of intersection points between cell boundary and 5 test rays from bottom to top.

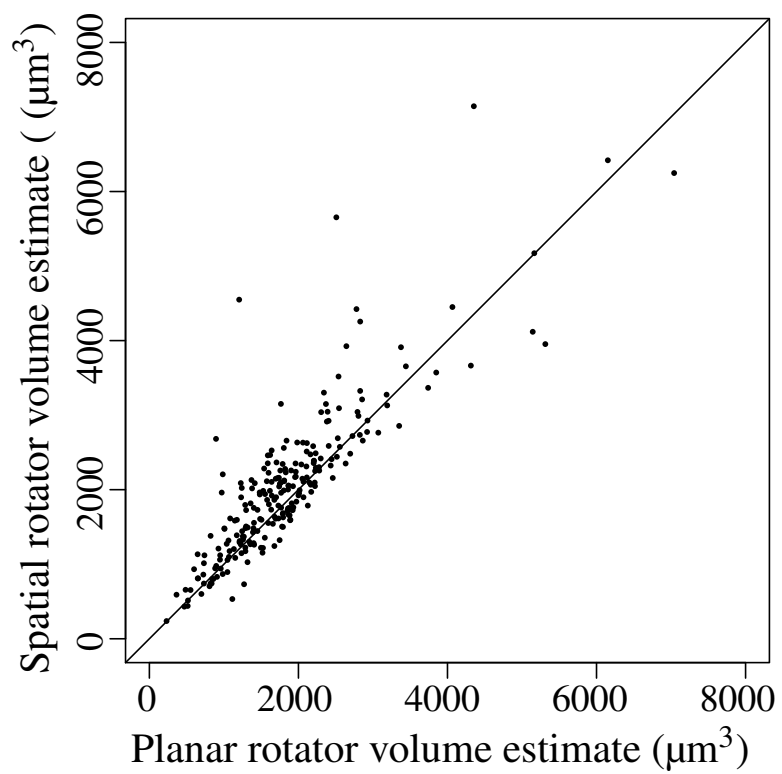

Figure 11: Spatial vs. planar rotator volume estimates using 5 test lines. For comparison, the identity line is also shown. 


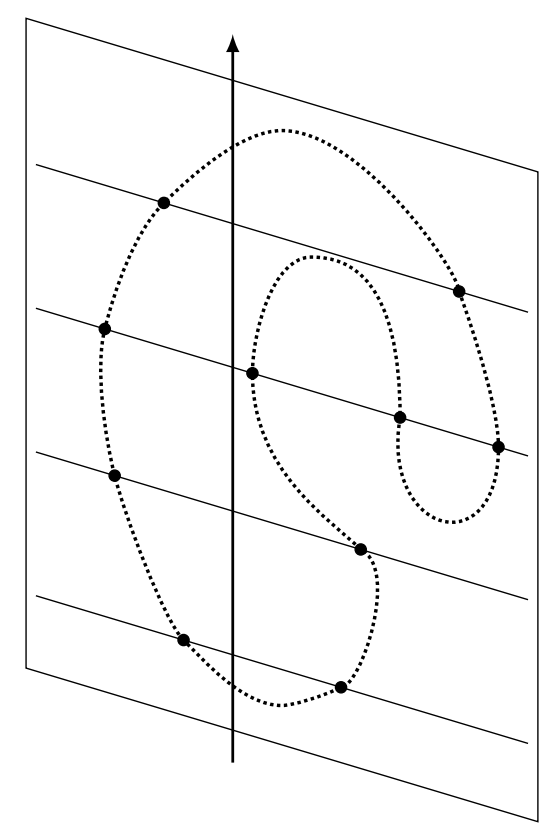

Figure 12: Particle with discrete vertical rotator probe. The profile of the particle on a vertical section is probed with $n=4$ systematic test lines perpendicular to the vertical axis.

\section{Discussion}

In the present paper, we have developed a new local volume estimator, the spatial rotator, based on a virtual 3D probe. In the extensive simulation study presented in Section 3, the spatial rotator was found to have lower variance than the planar rotator if at least 4 or 5 test rays were used. Furthermore, the spatial rotator can be used without physically rotating the tissue under study. It requires, however, that intersection points between test rays and cell boundaries can be determined accurately, also at the peripheral parts of the cell boundaries.

In Section 2, it has been shown that the spatial rotator can be regarded as a derivative of the Cavalieri estimator. The spatial rotator can also be regarded as a derivative of the discretized vertical rotator which was introduced in Jensen and Gundersen (1993) as an unbiased method of estimating particle volume from one vertical section. The discretized vertical estimator is based on measuring intersection lengths with a grid of parallel test lines perpendicular to the vertical axis as illustrated in Figure 12. The position of the test lines are systematically sampled in the same way as for the spatial rotator, but intersection distances are measured on two half lines each. Using a formula equivalent to (2.1) and averaging over the two half lines, these measurements yield the squared ray distance $\ell_{i}^{2}$ for the $i$-th pair of half lines. The estimator for the particle volume then takes the form (2.2). The spatial rotator can be interpreted as an average over $n$ vertical rotators using vertical sections with systematically sampled direction. On each section, only one half line is evaluated, and the positions of the half lines given by their intersection with the vertical axis form a systematic sample, see Figure 13. 


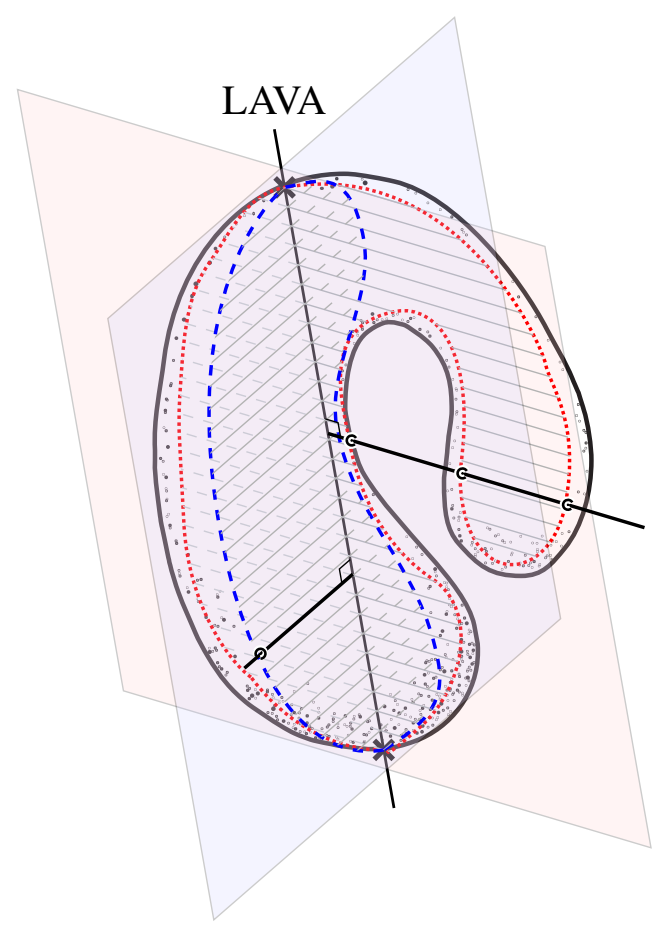

Figure 13: Interpretation of the spatial rotator as a vertical rotator probe. Two of the test rays from Figure 1 are shown here together with the corresponding vertical sections.

In the practical study, it was difficult to identify the top and bottom of the sampled cells. The largest radius $r$ from the nucleolus to the cell boundary, measured in the focal plane through the nucleolus, was used to estimate the top and bottom of the cell. The top and bottom were placed $r$ above and below the nucleolus. This procedure worked well in the practical study. In an adhoc manner, it was also tried to place the top and bottom at distance $f \cdot r$ where $f=0.5,0.75,1.5$ and 2.0. In these cases, the spatial rotator estimates were found to be biased by a factor proportional to $f$. These empirical findings emphasize the importance of being able to identify intersection points between test rays and cell boundaries accurately, also at the peripheral parts of the cells, if the spatial rotator is to be applied.

When evaluating the efficiency of a local volume estimator, it is important to take into account the workload involved in determining the estimator. The spatial rotator is inherently a fast probe as it requires minimum of manual workload. The number of intersection markings for each test ray besides marking of an interval on the LAVA is comparable to that of the planar rotator and nucleator. Since multiple focal planes are used, the time for the microscopic workstation to move between focal planes must however also be added to the total workload. This can be avoided by first recording focal planes and later perform the marking on an offline workstation.

Choosing between the spatial rotator and other Cavalieri estimators, in particular the point counting Cavalieri estimator, we recommend to use the spatial rotator when the particle profile areas on the various multiple intersection planes must be estimated manually. However, it is still unknown whether the spatial rotator is more efficient than other Cavalieri estimators when dealing with very complex particles. 


\section{Acknowledgements}

This work has been supported by The Danish National Advanced Technology Foundation, The Danish Council for Strategic Research and Centre for Stochastic Geometry and Advanced Bioimaging, funded by a grant from the Villum Foundation. Gratitude is also expressed to Bjarne Stig Andersen for the development of the rotus model for particle simulations.

\section{References}

B. B. Andersen, H. J. G. Gundersen, and B. Pakkenberg. Aging of the human cerebellum. Journal of Computational Neurology, 466:356-365, 2003.

A. J. Baddeley, Hans-Jørgen G. Gundersen, and Luis Manuel Cruz-Orive. Estimation of surface area from vertical sections. Journal of Microscopy, 142:259-276, 1986.

Adrian Baddeley and Eva B. Vedel Jensen. Stereology for Statisticians. Chapman \& Hall, Boca Raton, Florida, 2005.

Luis Manuel Cruz-Orive. Comparative precision of the pivotal estimators of particle size. Image Analysis and Stereology, 27:17-22, 2008.

Luis Manuel Cruz-Orive. The pivotal tesselation. Image Analysis and Stereology, 28:63-67, 2009.

Luis Manuel Cruz-Orive, ML Ramos-Herrera, and E. Aratacho-Pérula. Stereology of isolated objects with the invariator. Journal of Microscopy, 240:94-110, 2010.

Hans-Jørgen G. Gundersen. Stereology of arbitrary particles. A review of unbiased number and size estimators and the presentation of some new ones, in memory of William R. Thompson. Journal of Microscopy, 143:3-45, 1986.

Hans-Jørgen G. Gundersen. The nucleator. Journal of Microscopy, 151(1):3-21, 1988.

M. Hosseini-Sharifabad and J. R. Nyengaard. Design-based estimation of neuronal number and individual neuronal volume in the rat hippocampus. Journal of Neuroscience Methods, 162:206-214, 2007.

C. Hundahl, J. Kelsen, K. Kjaer, L. C. Ronn, R. E. Weber, E. Geuens, A. Hay-Schmidt, and J. R. Nyengaard. Does neuroglobin protect neurons from ischemic insult? A quantitative investigation of neuroglobin expression following transient MCAo in spontaneously hypertensive rats. Brain Research, 1085:19-27, 2006.

Eva B. Vedel Jensen. Local Stereology. World Scientific, Singapore, 1998.

Eva Bjørn Vedel Jensen and Hans-Jørgen G. Gundersen. The rotator. Journal of Microscopy, 170:35-44, 1993.

T. Mattfeldt, H.J. Möbius, and G. Mall. Orthogonal triplet probes: an efficient method for unbiased estimation of length and surface of objects with unknown orientation in space. Journal of Microscopy, 139:279-289, 1985. 
Jens R. Nyengaard and Hans-Jørgen G. Gundersen. The isector: a simple and direct method for generating isotropic, uniform random sections from small specimens. Journal of Microscopy, 165:427-431, 1992.

D. C. Sterio. The unbiased estimation of number and size of arbitrary particles using the disector. Journal of Microscopy, 134:127-136, 1984.

T. Tandrup, H. J. G. Gundersen, and E. B. V. Jensen. The optical rotator. Journal of Microscopy, 186(2):108-120, 1997.

\section{A Mathematical background for spatial rotator}

In this section, we give a short derivation of the estimation principle of the spatial rotator. Let $Y$ be a particle (bounded subset of $R^{3}$ ). The volume of $Y$ can be expressed by the intersection with a half plane $L_{2}^{+}(\alpha)$ rotated by an angle $\alpha$ around a fixed line $L_{1(0)}$, the LAVA. Letting $d\left(y, L_{1(0)}\right)$ denote the distance from $y$ to $L_{1(0)}$, we thus have

$$
\begin{aligned}
V(Y) & =\int_{0}^{2 \pi} \int_{Y \cap L_{2}^{+}(\alpha)} d\left(y, L_{1(0)}\right) \mathrm{d} y \mathrm{~d} \alpha \\
& =\int_{0}^{2 \pi} \int_{-\infty}^{\infty} \int_{0}^{\infty} d\left(\left(y_{1}, y_{2}, \alpha\right), L_{1(0)}\right) \mathbf{1}_{Y}\left(\left(y_{1}, y_{2}, \alpha\right)\right) \mathrm{d} y_{1} \mathrm{~d} y_{2} \mathrm{~d} \alpha,
\end{aligned}
$$

where $\left(y_{1}, y_{2}, \alpha\right)$ stands for the point with Cartesian coordinates $y_{1}, y_{2}$ on the half plane $L_{2}^{+}(\alpha)$. Now we use that the squared ray distance $\ell^{2}(p, \alpha ; Y)$ for a test ray in $L_{2}^{+}(\alpha)$, perpendicular to $L_{1(0)}$ and starting at $(0, p, \alpha) \in L_{1(0)}$, fulfills

$$
\ell^{2}(p, \alpha ; Y)=\int_{0}^{\infty} 2 d\left(\left(y_{1}, p, \alpha\right), L_{1(0)}\right) \mathbf{1}_{Y}\left(\left(y_{1}, p, \alpha\right)\right) \mathrm{d} y_{1},
$$

which gives us

$$
\begin{aligned}
V(Y) & =\int_{0}^{2 \pi} \int_{-\infty}^{\infty} \pi \ell^{2}(p, \alpha ; Y) \mathrm{d} p \frac{\mathrm{d} \alpha}{2 \pi} \\
& =\int_{\text {LAVA }} \int_{0}^{2 \pi} \pi \ell^{2}(p, \alpha ; Y) \frac{\mathrm{d} \alpha}{2 \pi} \mathrm{d} p,
\end{aligned}
$$

where "LAVA" means the minimal interval $\left[\mathrm{LAVA}_{0}, \mathrm{LAVA}_{1}\right]$ on $L_{1(0)}$ spanning the projection of $Y$ onto $L_{1(0)}$. The spatial rotator consists in estimating this integral by discretizing the test ray start points $p$ and their angles $\alpha$ simultaneously

$$
\hat{V}(Y)=d \pi \sum_{i=1}^{n} \ell^{2}\left(p_{i}, \alpha_{i} ; Y\right) .
$$

Here, $p_{i}=p_{1}+(i-1) d$ where $p_{1}$ is uniform in $[0, d]$ and $d=\left(\mathrm{LAVA}_{1}-\mathrm{LAVA}_{0}\right) / n$. Likewise, $\alpha_{i}=\alpha_{1}+(i-1) \vartheta$ where $\alpha_{1}$ is uniform in [0, $\left.\vartheta\right]$. Different choices of $\vartheta$ have been discussed in the main text of the paper. 